\title{
Análise de um método de debriefing para a simulação da ressuscitação cardiopulmonar: revisão integrativa
}

\author{
Analysis of a debriefing method for simulating cardiopulmonary resuscitation: an \\ integrative review
}

\begin{abstract}
Análisis de un método de debriefing para simular la reanimación cardiopulmonar: una revisión integradora
\end{abstract}

Juliana da Silva Garcia Nascimento ${ }^{1 *}$, Fabiana Cristina Pires², Kleiton Gonçalves do Nascimento², Daniela da Silva Garcia Regino ${ }^{3}$, Mateus Goulart Alves ${ }^{4}$, Jordana Luiza Gouvêa de Oliveira ${ }^{1}$, Tainá Vilhar Siqueira ${ }^{1}$, Maria Celia Barcellos Dalri'

\section{RESUMO}

Objetivo: Sintetizar os conteúdos necessários para planejar e executar o Structured and Supported Debriefing no processo de ensino e aprendizagem da ressuscitação cardiopulmonar em ambiente simulado. Métodos: Revisão integrativa da literatura, realizada em maio de 2021, nas fontes National Library of Medicine, Literatura Latino-Americana e do Caribe em Ciências da Saúde, Scopus, Cumulative Index to Nursing and Allied Health Literature e Web of Science, com auxílio do Rayyan para seleção dos artigos e adoção do referencial de Análise de Conteúdo. Resultados: Identificaram-se, a priori, 469 estudos e quatro compuseram a amostra final. Elaboraram-se duas categorias: critérios que compõem o método structured and supported debriefing, caracterizados pelos objetivos das fases do debriefing; as ações pertinentes a cada fase; as questões disparadoras do debriefing e o tempo de execução, e fatores que interferem na qualidade do debriefing structured and supported, principalmente a agressividade dos facilitadores do debriefing na abordagem dos estudantes e a ênfase nos erros cometidos durante a execução do cenário clínico. Considerações finais: Deve-se adotar os critérios do structured and supported debriefing para sustentar a qualidade do seu planejamento e execução frente ao processo de ensino e aprendizagem da ressuscitação cardiopulmonar em ambiente simulado.

Palavras-chave: Simulação, Reanimação cardiopulmonar, Ensino aprendizagem, Enfermagem.

\begin{abstract}
Objective: To synthesize the necessary contents to plan and execute structured and supported debriefing in the teaching and learning process of cardiopulmonary resuscitation in a simulated environment. Methods: Integrative review, carried out in May 2021, in the National Library of Medicine, Latin American and Caribbean Literature in Health Sciences, Scopus, Cumulative Index to Nursing and Allied Health Literature and Web of Science sources, with the assistance of Rayyan for selection of articles and adoption of the Content Analysis framework. Results: 469 studies were identified and four composed the final sample. Two categories were created: criteria for planning and executing the structured and supported debriefing method, characterized by objectives relevant to each phase of the debriefing; the actions that make it possible to achieve the goals;
\end{abstract}

\footnotetext{
${ }^{1}$ Universidade de São Paulo (USP), Ribeirão Preto - SP. *E-mail: mestradounesp28@yahoo.com.br

2 Universidade Federal do Triângulo Mineiro (UFTM), Uberaba - MG.

3 Universidade Brasil (UB), Fernandópolis - SP.

${ }^{4}$ Universidade de Franca (UNIFRAN), Franca - SP.
}

SUBMETIDO EM: 9/2021

ACEITO EM: 9/2021

PUBLICADO EM: 9/2021 
issues that trigger debriefing and execution time, and factors that interfere with the quality of structured and supported debriefing, mainly the aggressive approach to students and the emphasis on mistakes made. Final considerations: The structured and supported debriefing criteria must be adopted to support the quality of its planning and execution in the light of the teaching and learning process of simulating cardiopulmonary resuscitation.

Key words: Simulation, Cardiopulmonary resuscitation, Teaching learning, Nursing.

\section{RESUMEN}

Objetivo: Sintetizar los contenidos necesarios para planificar y ejecutar debriefing estructurado y apoyado en el proceso de enseñanza y aprendizaje de la reanimación cardiopulmonar en un entorno simulado. Métodos: Revisión integrativa, realizada en mayo de 2021, en Biblioteca Nacional de Medicina, Literatura Latinoamericana y del Caribe en Ciencias de la Salud, Scopus, Índice Acumulativo de Literatura en Enfermería y Afines y fuentes Web of Science, con asistencia de Rayyan para la selección de artículos y adopción del marco de Análisis de Contenido. Resultados: Se identificaron 469 estudios y cuatro compusieron la muestra final. Se crearon dos categorías: criterios para planificar y ejecutar el método de debriefing estructurado y apoyado, caracterizado por los objetivos relevantes para cada fase del debriefing; las acciones que permitan alcanzar las metas; problemas que desencadenan el debriefing y el tiempo de ejecución, y factores que interfieren con la calidad del debriefing estructurado y apoyado, principalmente el enfoque agresivo hacia los estudiantes y el énfasis en los errores cometidos. Consideraciones finales: Los criterios de debriefing estructurados y sustentados deben ser adoptados para apoyar la calidad de su planificación y ejecución a la luz del proceso de enseñanza y aprendizaje de la simulación de reanimación cardiopulmonar.

Palabras clave: Simulación, Reanimación cardiopulmonar, Enseñanza aprendizaje, Enfermería.

\section{INTRODUÇÃO}

Estudantes no âmbito da saúde, especialmente em enfermagem, são submetidos a diversos mecanismos pedagógicos que podem facilitar ou dificultar a aprendizagem (ANDERSON M, et al., 2019). Nessa perspectiva, a simulação clínica, caracterizada como uma estratégia ativa de ensino e aprendizagem que replica situações reais, em ambiente seguro e controlado, configura uma possibilidade educativa por auxiliar no enfrentamento das barreiras impostas pelos métodos tradicionais de ensino (ANDERSON M, et al., 2019; INTERNATIONAL NURSING ASSOCIATION FOR CLINICAL SIMULATION AND LEARNING, 2016; RIBEIRO LL, et al., 2021).

A simulação clínica compõe-se de três etapas: preparação, participação e debriefing. A etapa de preparação abrange a disponibilização para o aprendiz de referenciais que embasarão o seu estudo e do treinamento de habilidades, preparando-o para executar o cenário simulado. Na participação vivencia-se o cenário proposto, já o debriefing define-se por um processo analítico de discussão/reflexão, realizado geralmente, após o cenário, com a intenção de fomentar as habilidades cognitivas, psicomotoras e afetivas do aprendiz (TYERMAN J, et al., 2019).

Considera-se o debriefing um componente fundamental da simulação clínica, que pode ser responsável pela mais alta probabilidade de sucesso no desenvolvimento de competência clínica, caso seja utilizado um método adequado aos objetivos pretendidos na simulação (HALAMEK LP, et al., 2019).

Além da American Heart Association recomendar a inclusão do debriefing nos treinamentos voltados à Ressuscitação Cardiopulmonar (RCP), em 2009 uniu-se ao Winter Institute for Simulation, Education, and Research (WISER) para elaborar um método de debriefing apropriado para o ensino desta temática, denominado structured and supported debriefing e conhecido como G.A.S debriefing, devido aos acrônimos que representam suas fases: G- Gather; A-analyze e S- summarize (BHANJI F, et al., 2015). 
Na primeira fase do G.A.S debriefing (G- Gather) realiza-se a exploração dos sentimentos dos aprendizes sobre o cenário clínico, na segunda fase (A- Analyze) ocorre a articulação do que se vivenciou com os referenciais teóricos pertinentes a RCP e na última fase (S- Summarize) inclui-se o resumo das lições aprendidas (BHANJI F, et al., 2015; KIM JH, et al., 2017).

Apesar do G.A.S debriefing ser indicado como ideal para a simulação clínica da RCP devido ao fácil manuseio, até para facilitadores principiantes no debriefing, ainda não foram desenvolvidos estudos capazes de apresentar os conteúdos necessários para realizar o planejamento deste método, condição que pode interferir na qualidade do processo de ensino e aprendizagem da RCP em ambiente simulado (BHANJI F, et al., 2015; KIM JH, et al., 2017).

Diante da importância do ensino da RCP para estudantes no âmbito da saúde e para a segurança dos pacientes, e da premissa de que o G.A.S debriefing é o referencial teórico-metodológico mais indicado neste contexto, faz-se importante compreender os critérios que sustentam a sua execução, a fim de direcionar docentes e facilitadores na simulação clínica da RCP (BHANJI F, et al., 2015; KIM JH, et al., 2017).

Este estudo objetivou sintetizar os conteúdos necessários para planejar e executar o debriefing structured and supported no processo de ensino e aprendizagem da ressuscitação cardiopulmonar em ambiente simulado.

\section{MÉTODOS}

Trata-se de uma revisão integrativa de literatura, realizada em maio de 2021 em uma universidade pública do interior de São Paulo, Brasil, sustentada pela recomendação Preferred Reporting Items for Systematic Reviews and Meta-Analyses (PRISMA) (MOHER D, et al., 2010).

Cumpriram-se as etapas: (1) identificação do tema e seleção da questão de pesquisa; (2) estabelecimento de critérios para inclusão e exclusão de estudos e busca na literatura; (3) definição das informações extraídas dos estudos selecionados/categorização dos estudos; (4) avaliação dos estudos incluídos; (5) interpretação dos resultados; (6) apresentação da síntese do conhecimento (GANONG LH, 1987).

A estratégia Patient, Intervention, Comparison e Outcomes (PICO) foi utilizada para elaborar a questão de pesquisa, considerando-se como acrônimo $\mathrm{P}$ - população: estudantes na área da saúde; acrônimo I intervenção: a síntese dos conteúdos para planejar e executar o structured and supported debriefing e como acrônimo O - desfecho: o processo de ensino e aprendizagem da RCP em ambiente simulado. $\mathrm{O}$ acrônimo $\mathrm{C}$ não foi adotado para este estudo (SANTOS CM, et al., 2007). Desta forma, configurou-se a pergunta: Quais as evidências científicas disponíveis na literatura quanto aos conteúdos que sustentam o planejamento e a execução do método structured and supported debriefing para o processo de ensino e aprendizagem de estudantes em saúde da ressuscitação cardiopulmonar em ambiente simulado?

Foram incluídos estudos primários que respondessem à pergunta de pesquisa; sem delimitar idioma, com recorte temporal a partir de 2009, ano em o structured and supported debriefing foi recomendado para 0 ensino da RCP. Excluíram-se estudos do tipo revisão, teses, dissertações, artigos de opinião, comentários, ensaios, notas prévias e manuais. A busca das evidências foi compreendida em maio de 2021 nas fontes de

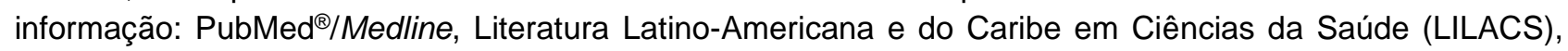
Scopus, Cumulative Index to Nursing and Allied Health Literature (CINAHL) e Web of Science.

Identificaram-se, na PubMed ${ }^{\circledR} /$ Medline e Scopus os descritores controlados em Medical Subjects Headings (MeSH) na língua inglesa: "Students, Health Occupations"; "Cardiopulmonary Resuscitation"; Learning; "Simulation Training" e a Palavra-chave: "Debriefing Structured and Supported". Adotou-se a estratégia: ("Students, Health Occupations" OR "Health Occupations Students" OR "Health Occupations Student" OR "Occupations Student, Health" OR "Occupations Students, Health" OR "Student, Health Occupations" AND "Debriefing Structured and Supported" AND "Cardiopulmonary Resuscitation" OR "Resuscitation, Cardiopulmonary" OR CPR OR "Cardio-Pulmonary Resuscitation" OR "Cardio Pulmonary Resuscitation" OR "Resuscitation, Cardio-Pulmonary" OR "Code Blue" OR "Mouth-to-Mouth Resuscitation" OR "Mouth to Mouth 
Resuscitation" OR "Mouth-to-Mouth Resuscitations" OR "Resuscitation, Mouth-to-Mouth" OR "Resuscitations, Mouth-to-Mouth" OR "Basic Cardiac Life Support" OR "Life Support, Basic Cardiac" AND Learning OR "Memory Training" OR "Training, Memory" AND "Simulation Training" OR "Training, Simulation" OR "Interactive Learning" OR "Learning, Interactive").

$\mathrm{Na}$ CINAHL adotaram-se os descritores controlados identificados em "Títulos/assunto" na língua inglesa: Students; "Cardiopulmonary Resuscitation"; Learning; "Simulation Training" e a Palavra-chave: "Debriefing Structured and Supported". A estratégia utilizada foi: (Students AND "Debriefing Structured and Supported" AND "Cardiopulmonary Resuscitation" AND Learning OR "Memory Training" OR "Training, Memory" AND "Simulation Training" OR “Training, Simulation" OR "Interactive Learning" OR "Learning, Interactive").

Na LILACS identificaram-se os descritores controlados nos Descritores em Saúde (DeCS), configurandose em Students; Cardiopulmonary Resuscitation; Learning; Simulation e a Palavra-chave: Debriefing Structured and Supported. A estratégia utilizada em inglês foi: (Students AND Debriefing Structured and Supported' AND Cardiopulmonary Resuscitation AND Learning AND Simulation). Em espanhol: (Estudiantes AND Debriefing Structured and Supported" AND Reanimación Cardiopulmonar AND Aprendizaje AND Simulación). Em português: (Estudantes AND Debriefing Structured and Supported" AND Reanimação Cardiopulmonar AND Aprendizagem AND Simulação).

$\mathrm{Na}$ Web of Science os descritores foram: Students, Health Occupations; Cardiopulmonary Resuscitation; Learning; Simulation Training e a Palavra-chave: Debriefing Structured and Supported. A estratégia adotada foi: $T S=$ Students ${ }^{*}$ Health Occupations ${ }^{*}$ AND Debriefing ${ }^{*}$ Structured and Supported ${ }^{*}$ AND Cardiopulmonary ${ }^{*}$ Resuscitation* AND Learning AND Simulation* Training)

Para selecionar os estudos, procedeu-se a leitura de títulos e resumos, com auxílio do aplicativo de revisão Rayyan, um programa virtual e gratuito que agiliza o processo de seleção de forma confiável por dois revisores independentes (OUZZANI M, et al., 2016).

As divergências entre suas avaliações resultaram em 11 estudos, enviados para um terceiro revisor, responsável por decidir a inclusão ou exclusão dos manuscritos. Após, realizou-se a leitura na íntegra dos estudos para definir a amostra final. A extração das informações pertinentes foi realizada por meio de um instrumento validado e adaptado para a presente pesquisa considerando-se: autores; ano de publicação; país de origem do estudo; objetivos; tipo de estudo; principais achados (URSI ES e GALVÃO CM, 2006). Por fim, classificou-se o nível de evidência dos manuscritos (GALVÃO CM, 2006).

Os achados foram analisados por meio da Análise Temática cumprindo-se três etapas: (1) pré-análise, configurada pela leitura flutuante das evidências e organização das informações convergentes; (2) exploração do material com agrupamento das convergências e (3) tratamento dos dados, determinando-se as categorias (MINAYO MC, 2017).

\section{RESULTADOS}

A seguir, têm-se o processo de seleção dos manuscritos incluídos na amostra da presente pesquisa, segundo o Preferred Reporting Items for Systematic Reviews and Meta-Analyses (PRISMA) (Figura 1) e a apresentação dos estudos, considerando-se autores, ano de publicação, país de origem, objetivos, tipo de estudo, principais resultados e nível de evidência (Quadro 1) (MOHER D, et al., 2010). 
Figura 1 - Fluxograma da seleção dos estudos adaptado do Preferred Reporting Items for Systematic Reviews and Meta-Analyses (PRISMA).

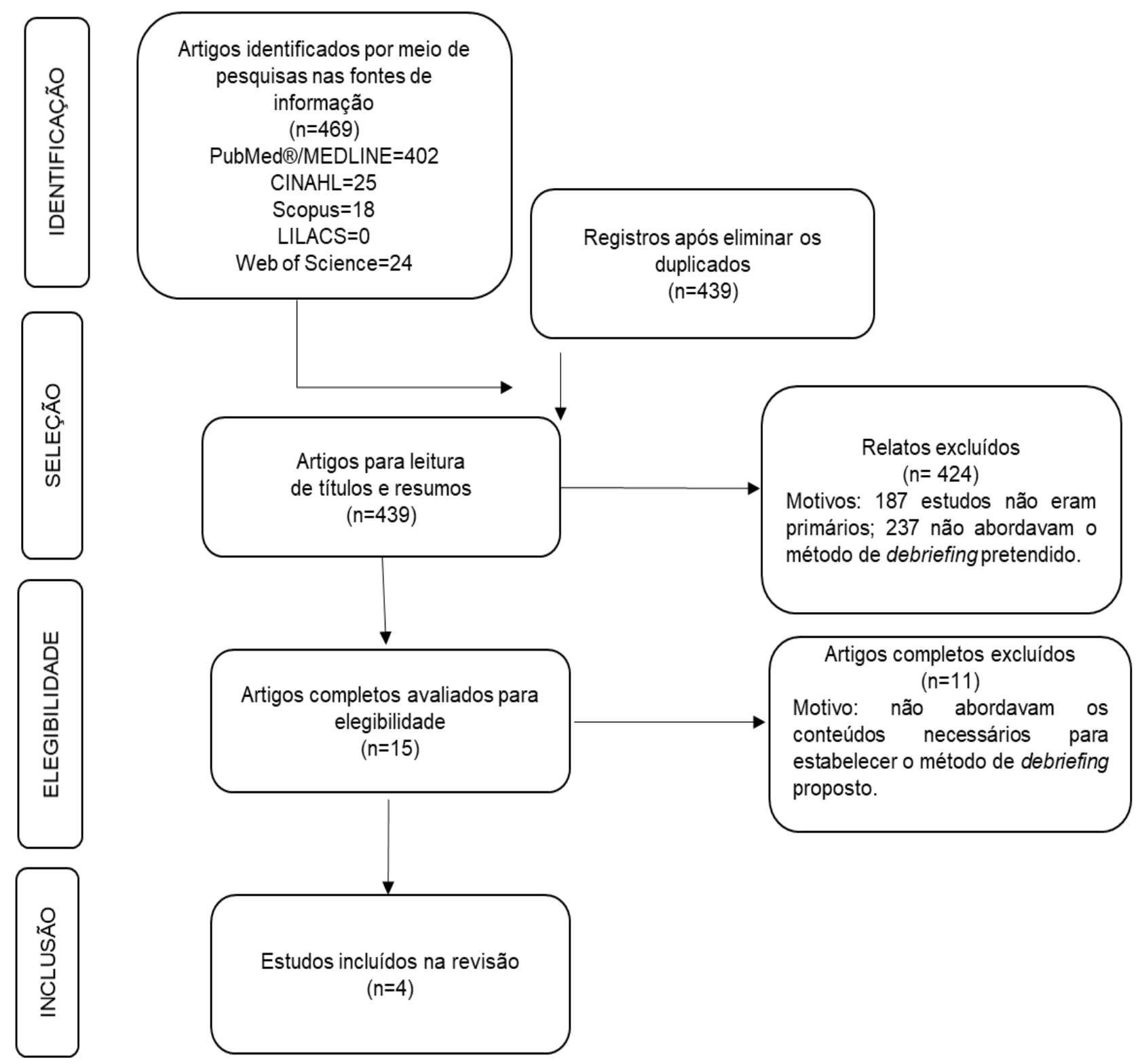

Fonte: NASCIMENTO JGS, et al., 2021. 
Quadro 1 - Caracterização dos estudos que abordaram o structured and supported debriefing.

\begin{tabular}{|c|c|c|}
\hline $\begin{array}{c}\text { Autor, ano, } \\
\text { país de origem }\end{array}$ & Objetivos e tipo de estudo & Principais achados e nível de evidência \\
\hline $\begin{array}{l}\text { KIM JH, et al., } \\
2017 . \\
\text { Coreia do Sul }\end{array}$ & $\begin{array}{l}\text { Comparar o impacto } \\
\text { educacional do feedback } \\
\text { versus structured and } \\
\text { supported debriefing na } \\
\text { simulação da ressuscitação } \\
\text { cardiopulmonar. Estudo } \\
\text { experimental, randomizado. }\end{array}$ & $\begin{array}{l}\text { A pontuação média do grupo submetido ao } \\
\text { structured and supported debriefing foi maior do que } \\
\text { o grupo que recebeu feedback para o conhecimento } \\
\text { e confiança [grupo controle: } 74,5(65,9-80,9) \text { - grupo } \\
\text { intervenção: } 85,0(71,9-87,6), p=0,035] \text {. Concluiu-se } \\
\text { que, o structured and supported debriefing provoca } \\
\text { um impacto positivo no processo de ensino e } \\
\text { aprendizagem desta temática. Nível de evidência } 2 \text {. }\end{array}$ \\
\hline $\begin{array}{l}\text { KIM M e KIM S, } \\
2017 . \\
\text { Coreia do Sul }\end{array}$ & $\begin{array}{l}\text { Identificar práticas de } \\
\text { debriefing no ensino de } \\
\text { enfermagem. Estudo descritivo } \\
\text { e exploratório. }\end{array}$ & $\begin{array}{c}\text { A adoção do structured and supported debriefing } \\
\text { pode tornar os facilitadores de uma simulação mais } \\
\text { aptos a apoiar as reações emocionais dos } \\
\text { estudantes. Concluiu-se que é necessário } \\
\text { desenvolver programas de treinamento sobre o } \\
\text { structured and supported debriefing. Nível de } \\
\text { evidência } 6 \text {. }\end{array}$ \\
\hline $\begin{array}{l}\text { BOWE S, et al., } \\
\quad 2017 . \\
\text { Estados Unidos }\end{array}$ & $\begin{array}{l}\text { Descrever métodos de } \\
\text { debriefing utilizados na } \\
\quad \text { aprendizagem da } \\
\text { ressuscitação cardiopulmonar. } \\
\text { Estudo descritivo exploratório. }\end{array}$ & $\begin{array}{l}\text { O instituto WISER colaborou com a American Heart } \\
\text { Association para elaborar o structured and supported } \\
\text { debriefing composto por três fases: reunir, analisar e } \\
\text { resumir. O componente suportado cria um ambiente } \\
\text { com apoio interpessoal e uso de protocolos para } \\
\text { apoiar o debriefing. O componente estruturado } \\
\text { embasa-se nas três fases citadas para orientar o } \\
\text { processo de reflexão. Nível de evidência } 6 \text {. }\end{array}$ \\
\hline $\begin{array}{l}\text { HUSEB } \varnothing \text { SE, et } \\
\text { al., } 2015 . \\
\text { Austrália }\end{array}$ & $\begin{array}{l}\text { Descrever a articulação do } \\
\text { ciclo de Gibbs com o } \\
\text { structured and supported } \\
\text { debriefing. Estudo descritivo- } \\
\quad \text { exploratório. }\end{array}$ & $\begin{array}{l}\text { O ciclo de aprendizagem reflexivo de Gibbs é um } \\
\text { modelo estruturado que pode apoiar as fases do } \\
\text { structured and supported debriefing. A articulação do } \\
\text { método structured and supported debriefing com as } \\
\text { etapas do ciclo de Gibbs é capaz de potencializar o } \\
\text { aprendizado. Nível de evidência } 6 \text {. }\end{array}$ \\
\hline
\end{tabular}

Fonte: NASCIMENTO JGS, et al., 2021.

Elaboraram-se duas categorias, a primeira denominada critérios que compõem o método structured and supported debriefing e a segunda intitulada fatores que interferem na qualidade do structured and supported debriefing.

A primeira categoria compôs-se de quatro critérios, caracterizados pelos objetivos das fases do método structured and supported debriefing; as ações que viabilizam as fases do método structured and supported debriefing; as questões disparadoras em cada fase e o tempo de duração das fases do structured and supported debriefing.

O Quadro 2 apresenta os critérios que compõem o método structured and supported debriefing, de acordo com cada fase. 
Quadro 2 - Fases do structured and supported debriefing e seus respectivos critérios.

\begin{tabular}{|c|c|c|c|}
\hline Critérios & $\begin{array}{c}\text { 10 fase: G- Gather: reunir } \\
\text { informações }\end{array}$ & $\begin{array}{l}2^{\circ} \text { fase: A- Analyze: } \\
\text { analisar informações }\end{array}$ & $\begin{array}{l}3^{\circ} \text { fase: S- Summarize: } \\
\text { resumir informações }\end{array}$ \\
\hline Objetivo & $\begin{array}{l}\text { Ouvir os participantes e } \\
\text { tranquilizar suas emoções }\end{array}$ & $\begin{array}{l}\text { Analisar as ações } \\
\text { realizadas e manter o } \\
\text { foco nos objetivos de } \\
\text { aprendizagem }\end{array}$ & $\begin{array}{l}\text { Facilitar a identificação e a } \\
\text { revisão das lições } \\
\text { aprendidas }\end{array}$ \\
\hline Ações & $\begin{array}{c}\text { Solicitar que os componentes da } \\
\text { equipe expressem o que pensam } \\
\text { e o que sentiram durante o } \\
\text { cenário }\end{array}$ & $\begin{array}{l}\text { Rever os acontecimentos; } \\
\text { relatar as observações } \\
\text { corretas e as que podem } \\
\text { melhorar }\end{array}$ & $\begin{array}{l}\text { Os participantes identificam } \\
\text { aspectos positivos de } \\
\text { comportamentos e os que } \\
\text { exigem mudanças. } \\
\text { Promove-se o resumo das } \\
\text { reflexões realizadas } \\
\text { durante o debriefing }\end{array}$ \\
\hline Questões & $\begin{array}{l}\text { Como vocês se sentem? você } \\
\text { pode nos dizer o que ocorreu } \\
\text { quando...? vocês podem } \\
\text { adicionar algum comentário sobre } \\
\text { esta situação? }\end{array}$ & $\begin{array}{l}\text { me conte mais sobre; } \\
\text { conte-me sobre o aspecto } \\
\text { "X" do cenário. }\end{array}$ & $\begin{array}{l}\text { Liste duas ações ou } \\
\text { eventos que foram } \\
\text { eficazes. }\end{array}$ \\
\hline Tempo & $25 \%$ do tempo do debriefing total & $\begin{array}{r}50 \% \text { do t } \\
\text { debriefir }\end{array}$ & $\begin{array}{l}25 \% \text { do te } \\
\text { debriefin }\end{array}$ \\
\hline Autores & $\begin{array}{c}\text { KIM JH, et al., 2017; KIM M e KIM } \\
\text { S, 2017; HUSEBØ SE, et al., } \\
2017\end{array}$ & $\begin{array}{l}\text { KIM JH, et al., 2017; KIM } \\
\text { M e KIM S, 2017; } \\
\text { HUSEB } \varnothing \text { SE, et al., } 2017\end{array}$ & $\begin{array}{c}\text { KIM JH, et al., 2017; KIM M } \\
\text { e KIM S, 2017; HUSEB } \\
\text { SE, et al., } 2017\end{array}$ \\
\hline
\end{tabular}

Fonte: NASCIMENTO JGS, et al., 2021.

A segunda categoria abordou os fatores que podem interferir na qualidade do método structured and supported debriefing, configurando-se pela realização de perguntas que induzam respostas e não sejam pautadas nos objetivos de aprendizagem; a desconsideração das percepções dos participantes; a demonstração de desrespeito diante dos comentários dos aprendizes; o excesso de crítica e agressividade na abordagem dos aprendizes pelos facilitadores; a não articulação dos acontecimentos com as diretrizes da RCP, a ênfase nos erros dos participantes; a realização de aula expositiva sobre o tema ao invés de valorizar a vivência; a ausência de especialização do facilitador no âmbito da RCP. Desta forma, a agressividade na abordagem aos alunos durante $O$ G.A.S debriefing e a ênfase nos erros cometidos, foram os fatores mais abordados (KIM JH, et al., 2017; BOWE S, et al., 2017; KIM M e KIM S, 2017; HUSEB $\varnothing ~ S E$, et al., 2015).

\section{DISCUSSÃO}

A importância de explorar o método G.A.S debriefing, principalmente no ensino em enfermagem da RCP é sustentada pela amostra incipiente de manuscritos que abordam o tema e também, pelo nível de evidência da maioria dos artigos caracterizados por estudos descritivos, possivelmente pela necessidade de se estabelecer um corpo de conhecimento capaz de fundamentar a prática do G.A.S debriefing, para somente depois desenvolver outros tipos de estudos, que intencionem comprovar a sua efetividade (BHANJI F, et al., 2015; KIM JH, et al., 2017; PHRAMPUS PE e O'DONNELL JM, 2013; COUPER K, et al., 2016; CAMANHO GL, 2009; DÍAZ-NARVÁEZ VP e CALZADILLA-NÚÑEZ, 2016; PALAGANAS JC, et al., 2016).

Desta forma, este estudo confere ineditismo a ciência da RCP e da saúde por apresentar os conteúdos necessários para planejar e executar o G.A.S debriefing, ainda não esclarecidos por outras pesquisas, possibilitar a elaboração de instrumentos que norteiem o debriefing na RCP, e ainda, por apresentar os fatores capazes de interferir na qualidade deste processo, guiando docentes e facilitadores da simulação clínica para as melhores condutas no debriefing (BHANJI F, et al., 2015; KIM JH, et al., 2017).

A primeira fase do G.A.S debriefing (G: Gather) objetiva o acolhimento dos aprendizes, redução de sua ansiedade, escuta ativa e organização das informações relatadas. Uma pesquisa descritiva, desenvolvida em uma universidade pública brasileira com 17 estudantes de enfermagem, identificou a percepção desta 
população quanto o debriefing e constatou que, os aprendizes se sentiram apoiados na primeira etapa do debriefing, por ter a liberdade de exporem sentimentos, sem julgamentos (COSTA RR, et al.,2019).

Considera-se a chave para o aprendizado, no G.A.S debriefing, a fase intermediária desse processo, (A: Analyze) em que se descreve as ações realizadas, pontos fortes e os pontos que necessitam melhorias, exigindo dos facilitadores a destreza e sensibilidade para valorizar os aspectos que merecem atenção articular a teoria com a vivência (KIM JH, et al., 2017; HALL K e TORI K, 2017).

O objetivo da última fase do G.A.S debriefing (S: Summarize) é organizar as lições que foram aprendidas e refletir sobre os impactos para prática real, conferindo se os objetivos de aprendizagem foram alcançados. Corrobora com esta afirmação, um estudo experimental, randomizado, realizado com 60 participantes sobre simulação em RCP, na Bélgica, que apontou a importância desta etapa para o desenvolvimento da autoeficácia e confiança do aprendiz frente a um atendimento de emergência (COPPENS I, et al., 2018).

As ações que compõem cada fase do structured and supported debriefing são possibilitadas pelas questões disparadoras deste método de debriefing. Considera-se como papel do facilitador a condução dos questionamentos durante o debriefing, que caracterizam "gatilhos disparadores de reflexão" e permitem ao aprendiz conferir significado ao aprendizado. Para cada fase do debriefing são realizados determinados questionamentos, com a intencionalidade de desenvolver conhecimentos, trabalho em equipe, comunicação, resolução de problemas, tomada de decisão, além de raciocínio crítico em um ambiente semelhante ao real (FERNANDES MT e ALVES CN, 2019).

O tempo dispensado para o structured and supported debriefing é um importante elemento para organização da reflexão, durante a simulação. As fases de Reunião e Resumo são mais curtas; já para a fase de Análise, dispensa-se um tempo maior, capaz de propiciar a articulação da teoria com o cenário vivenciado (KIM JH, et al., 2017).

Os principais fatores que podem interferir na qualidade do método structured and supported debriefing são a agressividade na abordagem dos aprendizes e a ênfase nos erros cometidos. Um estudo descritivo, canadense, realizado em 2018, forneceu uma síntese das situações que podem ocorrer durante a execução do debriefing e das estratégias para ajudar o docente a gerenciá-las, concluindo que, o debriefing é permeado pela exposição de emoções e até mal-entendidos e que, a primeira chave para o sucesso em situações difíceis de debriefing é a consciência das potenciais situações que podem ocorrer, na tentativa de evitá-las, capacitando o educador com os recursos necessários para contornar as ocorrências e não demonstrar animosidade, impaciência ou rispidez diante dos erros cometidos pelos aprendizes na simulação (GRANT VJ, et al., 2018).

Um debriefing ineficaz pode provocar efeitos negativos de aprendizagem e desenvolver habilidades inadequadas de raciocínio clínico, deficiências clínicas e julgamento e erros na prática real. Ao invés de se evidenciar os erros dos aprendizes deve-se estabelecer, de forma não punitiva, a reflexão das possibilidades de melhoria e das melhores ações durante a execução do cenário (LEE J, et al., 2020).

Desta forma, compreende-se que, é de suma importância considerar o preparo dos facilitadores, em uma simulação clínica, para conduzir o structured and supported debriefing, capazes de adotar estratégias para minimizar as intercorrências que advém da discussão sobre a vivência e evitar uma abordagem inadequada dos aprendizes e a ênfase em suas lacunas cognitivas, procedimentais e atitudinais (LEE J, et al., 2020; BHANJI F, et al., 2015; KIM JH, et al., 2017).

A principal limitação para desenvolver este estudo configurou-se pela incipiência de artigos científicos que abordam a utilização do método structured and supported debriefing. Sugere-se o desenvolvimento de outras investigações científicas neste âmbito, por meio de estudos bem delineados metodologicamente, sustentados por maiores níveis de evidência, e também, a que, a inclusão do G.A.S debriefing nas propostas curriculares de cursos de graduação em enfermagem, para fomentar o aperfeiçoamento das competências clínicas referentes a RCP. 


\section{CONSIDERAÇÕES FINAIS}

Os conteúdos necessários para planejar e executar o debriefing structured and supported no processo de ensino e aprendizagem da ressuscitação cardiopulmonar em ambiente simulado caracterizam-se pelos objetivos de cada fase do método, as ações que viabilizam as fases, as questões norteadoras pertinentes a cada momento da discussão/reflexão e o tempo ideal para proceder o debriefing. A agressividade na abordagem dos estudantes durante o debriefing structured and supported e a ênfase nos erros cometidos pelos aprendizes são os principais fatores que podem interferir na qualidade do método structured and supported debriefing.

\section{REFERÊNCIAS}

1. ANDERSON M, et al. Simulation in Advanced Practice Education: Let's Dialogue. Clinical Simulation in Nursing. 2019; 26: 81-5.

2. BHANJI F, et al. Part 14: Education: 2015. American Heart Association Guidelines Update for Cardiopulmonary Resuscitation and Emergency Cardiovascular Care. Circulation. 2015; 132(18Suppl2): S561-73.

3. BOWE S, et al. Facilitation and Debriefing in Simulation Education. Otolaryngologic Clinics of North America 2017; 50(5): 989-1001.

4. CAMANHO GL. Nível de evidência. Revista Brasileira de Ortopedia. 2009; 44(6): 1-2.

5. COPPENS I, et al. The effectiveness of crisis resource management and team debriefing in resuscitation education of nursing students: A randomised controlled trial. Clinical Simulation in Nursing. 2018; 27(1-2): 77-85.

6. COSTA RR, et al. Perceptions of nursing students on the structural dimensions of clinical simulation. Sci Med. 2019; 29(1): e32972.

7. COUPER K, et al. An evaluation of three methods of in-hospital cardiac arrest educational debriefing: The cardiopulmonary resuscitation debriefing study. Resuscitation. 2016; 105: 130-7.

8. DÍAZ-NARVÁEZ VP, CALZADILLA-NÚNEZ A. Artículos científicos, tipos de investigación y productividad científica en las ciencias de la salud. Revista Ciencias de la Salud. 2016; 14(1): 115-21.

9. FERNANDES MT, ALVES CN. Simulação como metodologia na formação de discentes em enfermagem no estágio final da graduação. Atas de Ciências da Saúde. 2019; 7: 115-25.

10. GALVÃO CM. Níveis de Evidência. Acta Paulista de Enfermagem. 2006; 19(2): V.

11. GANONG LH. Integrative reviews of nursing research. Research in Nursing \& Health. 1987; 10(1): 1-11.

12. GRANT VJ, et al. Difficult debriefing situations: A toolbox for simulation educators. Medical Teacher. 2018; 40(7): 703712.

13. HALAMEK, LP, et al. Using briefing, simulation and debriefing to improve human and system performance. Seminars in Perinatology. 2019; 43(8): 151178.

14. HALL K, TORI K. Best practice recommendations for debriefing in simulation-based education for australian undergraduate nursing students: an integrative review. Clinical Simulation in Nursing. 2017; 13(1): 39-50.

15. HUSEB $\varnothing$ SE, et al. Reflective practice and its role in simulation. Clinical Simulation in Nursing. 2015; 11(8): $368-375$.

16. INTERNATIONAL NURSING ASSOCIATION FOR CLINICAL SIMULATION AND LEARNING (INACSL). INACSL Standards Committee. INACSL standards of best practice: Simulation ${ }^{S M}$ simulation-enhanced interprofessional education (sim-IPE). Clinical Simulation in Nursing. 2016; 12(S): S34-S38.

17. $\mathrm{KIM} \mathrm{JH}$, et al. Focused and Corrective Feedback Versus Structured and Supported Debriefing in a Simulation-Based Cardiac Arrest Team Training. Simulation in Healthcare. 2017; 12(3): 157-64.

18. KIM M, KIM S. Debriefing Practices in Simulation-Based Nursing Education in South Korea. Clinical Simulation in Nursing. 2017; 13: 201-9.

19. LEE J, et al. Debriefing methods and learning outcomes in simulation nursing education: A systematic review and meta-analysis. Nurse Education Today. 2020; 104345.

20. MINAYO MC. Sampling and saturation in qualitative research: consensuses and controversies. Revista Pesquisa Qualitativa. 2017; 5(7): 1-12.

21. MOHER D, et al. Preferred reporting items for systematic reviews and meta-analyses: the PRISMA statement. International Journal of Surgery Open. 2010; 8(5): 336-41.

22. OUZZANI M, et al. Rayyan-a web and mobile app for systematic reviews. Systematic Reviews. 2016; 5(1): 210.

23. PALAGANAS JC, et al. Structured debriefing in simulation-based education. AACN Advanced Critical Care. 2016; 27(1): 78-85.

24. PHRAMPUS PE e O'DONNELL JM. Debriefing using a structured and supported approach. In: LEVINE AI, et al. The Comprehensive Textbook of Healthcare Simulation. New York: Springer Science; 2013. p. 74-7.

25. RIBEIRO LL, et al. O uso da metodologia ativa como ferramenta de fortalecimento para a segurança do paciente. Revista Eletrônica Acervo Enfermagem. 2021; 8: e4889.

26. SANTOS CM, et al. A estratégia PICO para a construção da pergunta de pesquisa e busca de evidência. Revista Latino-Americana de Enfermagem. 2007; 15(3): 508-11.

27. TYERMAN J, et al. A systematic review of health care presimulation and briefing effectiveness. Clinical Simulation in Nursing. 2019; 27: 12-25.

28. URSI ES, GALVÃO CM. Prevenção de lesões de pele no perioperatório: revisão integrativa da literatura. Revista Latino-Americana de Enfermagem. 2006; 14(1): 124-31. 